\title{
Effect of Hyaluronan Enriched Transfer Medium on Conception Rate in Bovine Embryo Transfer
}

\author{
D. Reena ${ }^{1}$, R. Thirupathi ${ }^{2}$, S. Manokaran ${ }^{3 *}$, S. Rangasamy ${ }^{4}$, \\ S. Balasubramanian ${ }^{5}$ and A. Palanisammi ${ }^{6}$ \\ ${ }^{1}$ Department of Clinics, Madras Veterinary College, Chennai-600 007, India \\ ${ }^{2}$ Cattle Breeding and Fodder Development, Dharmapuri- 636705, India \\ ${ }^{3}$ Kangayam Cattle Research Station, Uppupallam, Baguthampalayam, Sathyamangalam, \\ Erode Dt. - 638 451, India \\ ${ }^{4}$ Department of Veterinary Gynaecology and Obstetrics, Madras Veterinary College, \\ Chennai - 600 007, India \\ ${ }^{5}$ Tamil Nadu Veterinary and Animal Sciences University, Madras Veterinary College campus, \\ Chennai - 600 007, India \\ ${ }^{6}$ Veterinary College and Research Institute, Tirunelveli - 627 358, India \\ *Corresponding author
}

\begin{abstract}
A B S T R A C T
Keywords

Bovines,

Recipients,

Synchronisation,

Hyaluronan,

Embryo transfer

Article Info

Accepted:

15 March 2020

Available Online:

10 April 2020

The present study was conducted to assess the effect of hyaluronan (HA) supplementation in the embryo transfer medium of in vivo produced, vitrified and warmed embryos on conception rate in bovines. The study was conducted in 12 recipient cows which were randomly divided into two groups viz. Group I (embryo transfer media with hyaluronan, experimental group) and Group II (embryo transfer media without hyaluronan, control group). The vitrified and warmed embryos were suspended in the embryo transfer medium according to the group, cultured and transferred to the synchronized recipients. The pregnancy diagnosis after 60 days revealed 50 and 33.33 per cent conception rate in Group I and II recipients, respectively. From the study it is evident that the enrichment of hyaluronan in the embryo transfer medium has beneficial effect on enhancing the conception rate in bovines.
\end{abstract}




\section{Introduction}

The media used for embryo collection/culture, handling, vitrification and embryo transfer play a major role in the conception rate of in vivo or in vitro produced frozen thawed embryos and researchers are still under exploration of suitable media to facilitate better fertility. To improve the conception rate, the media used for embryo transfer are usually supplemented with various protein sources.

One of the most widely used protein supplement in the media is serum but reports indicated that serum reduces the developmental potential of embryos (Bavister, 1995) and increases the sensitivity of embryos to cryopreservation and compromises viability of these embryos, as indicated by the rates of embryo survival after thawing (Vajta et al., 1997; Ohboshi et al., 1998) and pregnancy rates (Massip et al., 1995).

The embryo transfer media should mimic in vivo conditions and create an environment similar to that of the female reproductive tract. However, the protein supplemented media are not similar to the fluid composition of the female reproductive tract. Hyaluronan, a glycosaminoglycan is found at higher concentration in the follicular, oviductal and uterine fluids (Lane et al., 2003).

Hyaluronan possess properties like viscosity, lubrication, cell adhesion, migration, regulation of protein secretion, gene expression and cell proliferation that are responsible for initial attachment of embryos to endometrium facilitating implantation (Stojkovic et al., 2002). For these reasons, the present study was conducted to find the effect of hyaluronan on conception rate when it is supplemented in the embryo culture and transfer media of in vivo produced, vitrified and warmed bovine embryos.

\section{Materials and Methods}

A total of twelve non-pregnant cows with a postpartum interval of 90-120 days were selected from organized dairy farms and used as recipients in the study. All the recipients were between $2^{\text {nd }}$ to $4^{\text {th }}$ gestation, apparently healthy, regularly cycling and weighing between 300-325 kgs. The recipients were having normal fertility, healthy reproductive tract with normal ovaries and free from peri and postpartum complications during last calving. The selected cows were randomly divided in to two groups viz. Group I (with hyaluronan in the post warming culture and transfer media, $n=6$ ) and Group II (without hyaluronan in the post warming culture and transfer media, $\mathrm{n}=6$ ).

All the selected recipient cows were synchronized using the intra vaginal progesterone insert and prostaglandin $\mathrm{F}_{2} \alpha$ $\left(\mathrm{PGF}_{2} \alpha\right)$. These cows were inserted with progesterone device $\left(\right.$ TRIU-B ${ }^{\circledR}, \quad$ Virbac Animal Health Ltd., Argentina) between day 8 to 12 of the oestrous cycle. On day 9 , the cows were injected with $500 \mu \mathrm{g}$ Cloprostenol (Pragma $^{\mathrm{TM}}$, Intas Pharmaceuticals Ltd., Ahmedabad, India) and TRIU-B was withdrawn on day 10 of insertion. Immediately after removal of TRIU-B, the cows were observed for signs of behavioural estrus at least four times daily for a minimum of 30 minutes at each session combined with rectal palpation. After identifying the estrum, the embryo transfer was carried out of day 7 of estrum.

Vitrified embryos were warmed as described by Vajta et al (2000). All warming procedures were performed at $38.5^{\circ} \mathrm{C}$ on the warming stage fixed on the stereozoom microscope. The warming solution (WS) was tissue culture medium containing various concentrations of sucrose. Vitrified embryos were transferred for 5 minutes to warming 
solution 1 containing $0.5 \mathrm{M}$ sucrose dissolved in tissue culture media and supplemented with 10 per cent fetal bovine serum. The embryos were then transferred to warming solution 2 containing $0.25 \mathrm{M}$ sucrose dissolved in tissue culture media and supplemented with 10 per cent fetal bovine serum. The embryos were evaluated after warming and only grade 1 (Excellent) and grade 2 (Good) quality embryos were used for embryo transfer. Graded embryos were transferred into a well containing pre-equilibrated hyaluronan rich transfer medium $(0.5 \mathrm{mg} / \mathrm{ml}$, GLR 002: R\&D system) for $4 \mathrm{hrs}$ at $37^{\circ} \mathrm{C}$ in 5 per cent $\mathrm{CO}_{2}$ on the day of transfer. Embryos were loaded into $0.25 \mathrm{~mL}$ French straws in pre-equilibrated hyaluronan rich transfer medium. Straws containing embryos were loaded and transferred to recipients uterine horn on the ipsilateral side of the corpus luteum.

Pregnancy diagnosis was carried out on day 30 by ultrasonographic examination and day 60 by rectal palpation. Rectal palpation was repeated on day 90 and 150 of gestation for further confirmation. Statistical analysis of the data was carried out as per standard procedures outlined by Snedecor and Cochran (1994).

\section{Results and Discussion}

In this study, all the recipient cows (100 per cent) of group I and II had 100 per cent retention of the intra vaginal progesterone insert from the time of insertion to withdrawal. None of the cows had any complication during the synchronization period. Similar percentages of retention rate with intra vaginal insert were documented by Bhoraniya et al., (2012).

All the cows of group I and II exhibited estrus (100 per cent) following the synchronization of estrus protocol. This result clearly indicated that all the protocol applied for synchronization of estrus was highly effective to induce 100 per cent estrus in postpartum cows for embryo transfer. This was in accordance with the findings of Zaabel et al (2009) and Ganesh (2013).

In this study, the mean $( \pm S E)$ time for onset of induced estrus ranged from $40.05 \pm 1.29$ to $44.70 \pm 1.17$ hours. The earlier onset of oestrus observed in this study when compared to other findings might be due to the early accelerated production of oestradiol after PIVD withdrawal as described by Odde (1990) in CIDR protocols and proper observation of the cows for detection of oestrus. Even though mild variation was present between cows in the onset of estrum, statistically no significant difference $(\mathrm{P} \geq 0.05)$ was observed in the mean $( \pm \mathrm{SE})$ onset of induced estrus among them. It might be due to the fact that the $\mathrm{PGF}_{2} \alpha$ injection one day prior to TRIU-B withdrawal could result in complete luteolysis in all the experimental cows (Schmitt et al., 1996)

In the present study, the conception rate was higher (50 per cent) in experimental group (group I) than in control group (33.33 per cent). In the experimental group the hyaluronan was added in the post thaw culture and transfer medium. Hyaluronan is a biological polymer. When hyaluronan is added to culture medium, it enhances the developmental competence thereby improving the embryo blastocyst rates. When used during cryopreservation, post thaw reexpansion and hatching rates increased (Stojkovic et al., 2002 and Lane et al., 2003). Another use for hyaluronan is in embryo culture (Furnus et al., 1998). Concentrations of hyaluronan in cumulus cells of developing oocytes were correlated with normal embryo development thus facilitating its use in culture medium (Ball et al., 1982; Tirone et al., 1997). When used as a replacement for protein in culture media for bovine in vitro 
produced embryos, blastocyst development, total trophectoderm and cell counts were significantly higher than for embryos cultured in media containing bovine serum albumin (BSA) (Furnus et al., 1998; Stojkovic et al., 2002). Additionally, these embryos when cultured in hyaluronan prior to freezing showed lower cell damage post-thawing as compared to those cultured in BSA. Interestingly, when hyaluronan was combined with recombinant albumin and citrate during culture of bovine in vitro fertilized (IVF) embryos, post-thaw hatching rates were significantly higher than those cultured in BSA or polyvinyl alcohol, respectively (Lane et al., 2003).

Zho et al., (2013) demonstrated that primary trophoblasts produce high level of hyaluronan which enhances trophoblast proliferation and invasiveness and inhibits apoptosis through PI3K/AKT and MAPK/ERK1/2 signal pathways. They also indicated that hyaluronan plays an important role in the implantation, placentation and maintenance of normal pregnancy. The results of the present study was concurred with the study of Simon et al (2003) who reported higher conception rate, implantation and ongoing pregnancy rates in hyaluronic acid supplemented transfer medium when compared with albumin supplemented transfer medium. Gardner et al (1999) reported significantly higher implantation and fetal development rates in mouse when hyaluronan alone supplemented transfer medium than in hyaluronic acid and BSA supplemented transfer medium. The use of hyaluronan in the transfer medium may offer several advantages in the implantation process, such as a significant increase in both implantation and fetal development rates. Concern has been expressed about the immediate or late expulsion of embryos after their transfer to the uterine cavity (Mansour and Aboulghar, 2002). However, our experience, as well as that of other researchers (Gardner et al., 1999), suggests that the implantation and pregnancy rates were higher in the hyaluronan treatment group.

From the study it is concluded that hyaluronan can be successfully used to replace serum or bovine serum albumin in the medium as a protein source and hyaluronan can produce higher conception rate when used in embryo culture and transfer media of vitrified and warmed bovine embryos.

\section{References}

Ball, G.D., M.E. Bellin, R.L. Ax and First, N.L. 1982. Glycosaminoglycans in bovine cumulus-oocyte complexes: morphology and chemistry. Mol. Cell. Endocrinol. 28: 113 - 122.

Bavister, B.D., 1995. Culture of preimplantation embryos: facts and artifacts. Human Reproduction Update. 1: 91 - 148

Bhoraniya, H.L., A.J. Dhami, M. Naikoo, B.C. Parmar and Sarvaiya, N.P. 2012. Effect of oestrus synchronization protocols on plasma progesterone profile and fertility in post-partum anoestrus Kankrej cows. Trop. Anim. Health Prod., 44: online, DOI 10.1007/s11250-011-0057-1.

Ganesh, K., 2013. Conception rate following oestrus induction with CIDR in buffaloes treated for retained fetal membranes. M.V.Sc., thesis submitted to the TANUVAS, Chennai.

Gardner, D.K., H. Rodrigez-Martinez and Lane, M. 1999. Fetal development after transfer is increased by replacing protein with the glycosaminoglycan hyaluronan for mouse embryo culture and transfer. Human Reproduction. 14: 2575 - 2580.

Furnus, C.C., D.G. de Matos and Martinez, A.G. 1998. Effect of hyaluronic acid on development of in vitro produced bovine embryos. Theriogenology. 49: 1489 - 1499.

Lane, M., J.M. Maybach, K. Hooper, J.F. Haslerv and Gardner, D.K. 2003. Cryo survival and development of bovine 
blastocysts are enhanced by culture with recombinant albumin and hyaluronan. Mol. Reprod. Dev. 64: 70 - 78.

Mansour, R.T. and Aboulghar, M.A. 2002. Optimizing the embryo transfer technique. Hum Reprod. 17(5): 1149-1153.

Massip, A., P. Mermillod and Dinnyes, A. 1995. Morphology and biochemistry of in vitro produced bovine embryos: implications for their cryopreservation. Human Reproduction. 10: 3004 - 3011

Odde, K.H., 1990. Bacterial floras of the uterus of cows after calving on two hygienically contrasting farms. Vet. Rec. 128: 440 - 442.

Ohboshi, S., N. Fujihara, T. Yoshida and Tomagane, H. 1998. Ultrastructure of bovine in vitro produced blastocysts cryopreserved by vitrification. Zygote. 6: $17-26$.

Schmitt, E.J.P., T.C. Diaz, C.M. Barros, R.L. de la Sota, M. Drost, E.W. Fredriksson, C.R. Staples, R. Thorner and Thatcher, W.W. 1996. Differential response of the luteal phase and fertility in cattle following ovulation of the first-wave follicle with human chorionic gonadotropin or an agonist of gonadotropin-releasing hormone. J. of Anim. Sci. 74: 1074 - 1083.

Simon, M.D., A. Safran, A. Revel, E. Aizenman, B. Reubinoff and Porat-Katz, A. 2003. Hyaluronic acid can successfully replace albumin as the sole molecule in a human embryo transfer medium. Fertil. Steril. 79: 1434 - 1438.

Snedecor, G.M. and Cochran, W.C. 1994. Statistical Methods. $9^{\text {th }}$ edn., Oxford and IBM Publishing Company. Mumbai, India.

Stojkovic, M., S. Kolle, S. Peinl, P. Stojkovic,
V. Zakhartchenko and Thompson, J.G. 2002. Effects of high concentrations of hyaluronan in culture medium on development and survival rates of fresh and frozen thawed bovine embryos produced in vitro. Reproduction. 124: 141-153.

Tirone, E., C. D'Alessandris, V.C. Hascall, G. Siracusa. and Salustri, A. 1997. Hyaluronan synthesis by mouse cumulus cells is regulated by interactions between folliclestimulating hormone (or epidermal growth factor) and a soluble oocyte factor (or transforming growth factor beta1). J. Biol. Chem. 272: 4787 - 4794.

Vajta, G., P. Hyttel and Callesen, H. 1997. Morphological changes of in vitroproduced bovine blastocysts after vitrification, in straw direct rehydration, and culture. Mol. Reprod. Dev. 48: 9-17.

Vajta,G., P. Holm, M. Kuwayama, P.J. Booth, H. Jacobssen, T. Greve and H. Callesen (1998). Open pulled straw (OPS) vitrification: A new way to reduce cryoinjuries of bovine ova and embryos. Molecular Reproduction and Development. 51: 53-58

Zaabel, S.M., A.O. Hegab, A.E. Montasser and El-Sheikh, H. 2009. Reproductive performance of anestrus buffaloes treated with CIDR. Anim. Prod. 6: 460 - 464.

Zhu, R., Y.H. Huang, Y. Tao, S.C. Wang, Ch. Sun, H.L. Piao, X.Q. Wang, M.R. Du and $\mathrm{Li}$, D. 2013. Hyaluronan up-regulate growth and invasion of trophoblasts in an autocrine manner via P13K/AKT and MAPK/ERK1/2 pathways in early human pregnancy. Placenta 34: 784-791.

\section{How to cite this article:}

Reena, D., R. Thirupathi, S. Manokaran, S. Rangasamy, S. Balasubramanian and Palanisammi, A. 2020. Effect of Hyaluronan Enriched Transfer Medium on Conception Rate in Bovine Embryo Transfer. Int.J.Curr.Microbiol.App.Sci. 9(04): 1841-1845. doi: https://doi.org/10.20546/ijcmas.2020.904.215 